\title{
Medical Image of the Month: Renal Cell Carcinoma with Extensive Tumor Thrombus
}

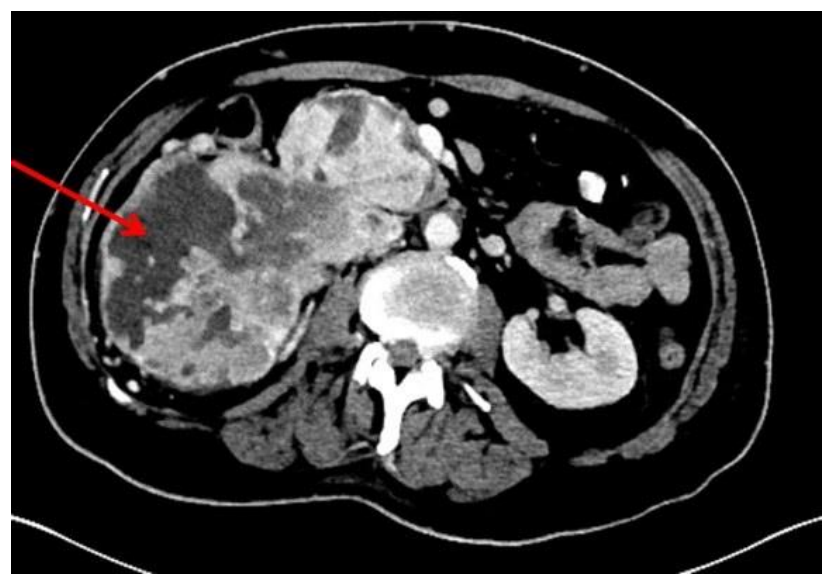

Figure 1. CT of the abdomen with contrast (axial image) shows a large right large heterogeneous mass (red arrow), consistent with renal cell carcinoma.

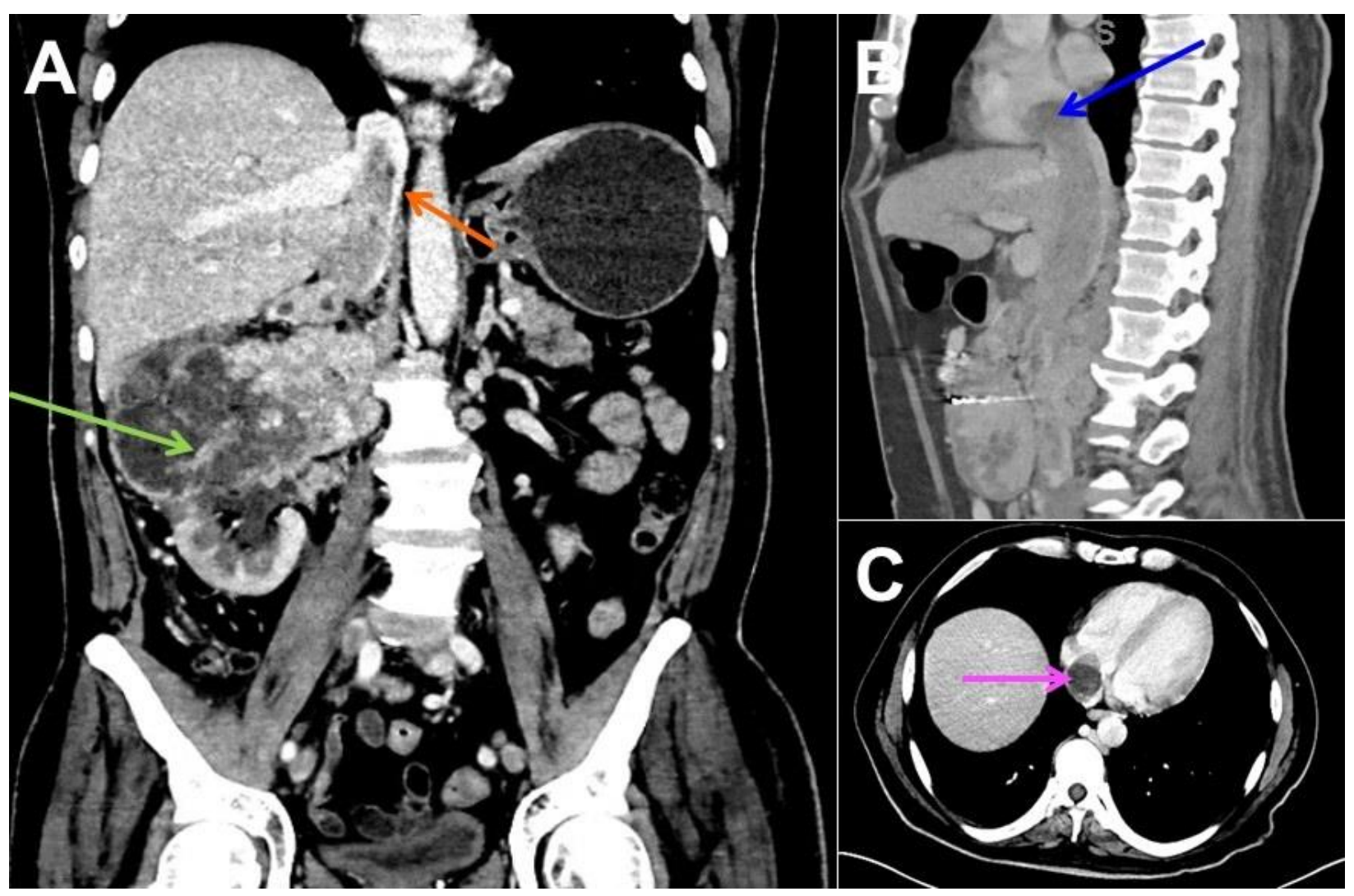

Figure 2. A: CT of the abdomen with contrast (coronal image) shows a large right renal mass (green arrow) and tumor thrombus in the IVC (orange arrow). B: Sagittal image showing extension of the tumor thrombus from the inferior vena cava into the right atrium (blue arrow). C: Axial image showing evidence of tumor thrombus in the right atrium (pink arrow). 
A 53-year-old man with a right-sided renal cell carcinoma (RCC) presented with nausea, vomiting, intolerance of oral intake and melena. A contrast enhanced CT of the abdomen and pelvis showed near complete replacement of the right kidney by a large heterogeneous mass, measuring $10 \times 16 \mathrm{~cm}$ (Figure 1). The mass invaded the renal vein and inferior vena cava (IVC) with extension to the level of the inferior cavo-atrial junction (Figure 2). The mass compressed the duodenum, causing a bowel obstruction. Liver and lung metastases were also found. A duodenal stent was placed with significant improvement in his nausea and vomiting. He was not able to receive anticoagulation due to severe gastrointestinal bleeding. The patient discontinued disease modifying therapy and died four weeks after discharge from the hospital.

Tumor thrombus occurs when a tumor invades a blood vessel. It occurs in approximately $10 \%$ of patients with renal cell carcinoma, which is a highly vascular malignancy with a propensity to invade the venous system (1). Extension of the tumor from the inferior vena cava into the right atrium is very uncommon, seen in only about $1 \%$ of RCCs (1). The American Joint Committee on Cancer staging system for RCC differentiates between tumor thrombus involving the renal vein (T3a), IVC below the diaphragm (T3b) and IVC above the diaphragm (T3c) (1). The presence of tumor thrombus changes staging, prognosis and surgical options. Surgical treatment may be the approach to tumor thrombus in RCC without metastatic disease. The surgical approach is often complex and requires extensive surgical planning and expertise (2). Perioperative morbidity and mortality appear to be proportional to the height of tumor growth, and tumor thrombus extending above the diaphragm carries increased perioperative risk. Wagner et al. (3) retrospectively studied 1,192 cases, and found reduced long-term survival in patients with any venous involvement. However, they found no significant difference in long-term survival between patients with IVC tumor thrombus below (T3b) or above (T3c) the diaphragm. In this study, the most important prognostic factors in RCC included renal tumor size, the presence of perinephric fat invasion, lymph node involvement and distant metastatic lesions.

David Horn MD, Sue Cassidy ANP-BC and Linda Snyder MD Departments of Internal Medicine and Pulmonary, Critical Care, Allergy and Sleep Medicine University of Arizona College of Medicine Tucson, AZ USA

\section{References}

1. Wotkowicz C, Wszolek MF, Libertino JA. Resection of renal tumors invading the vena cava. Urol Clin N Am. 2008; 35: 657-71. [CrossRef] [PubMed]

2. Quencer KB, Friedman T, Sheth R, Rahmi O. Tumor thrombus: incidence, imaging, prognosis and treatment. Cardiovasc Diagn Ther. 2017;7(Suppl 3):S165-77. [CrossRef] [PubMed]

3. Wagner B, Patard JJ, Méjean A, et al. Prognostic value of renal vein and inferior vena cava involvement in renal cell carcinoma. Eur Urol. 2009;55:452-9. [CrossRef] [PubMed] 